\title{
On certain sums over ordinates of zeta-zeros II
}

\author{
Andriy Bondarenko, Aleksandar Ivić, Eero Saksman and Kristian Seip
}

Dedicated to the memory of S. Srinivasan (1943 - 2005)

\begin{abstract}
Let $\gamma$ denote the imaginary parts of complex zeros $\rho=\beta+i \gamma$ of $\zeta(s)$. The problem of analytic continuation of the function $G(s):=\sum_{\gamma>0} \gamma^{-s}$ to the left of the line $\Re s=-1$ is investigated, and its Laurent expansion at the pole $s=1$ is obtained. Estimates for the second moment on the critical line $\int_{1}^{T}\left|G\left(\frac{1}{2}+i t\right)\right|^{2} \mathrm{~d} t$ are revisited. This paper is a continuation of work begun by the second author in [Iv01].
\end{abstract}

2010 Mathematics Subject Classification. 11M06

Keywords. Riemann zeta-function, Riemann hypothesis, analytic continuation, Laurent expansion, second moment

\section{Introduction: the function $G(s)$}

We will be concerned with the Dirichlet series

$$
G(s):=\sum_{\gamma>0} \gamma^{-s}
$$

where $\gamma$ denotes the ordinates of the complex zeros of the Riemann zeta-function $\zeta(s)$, counted as usual with multiplicities. Thus $G(s)$ converges absolutely and represents a holomorphic function in the half-plane $\sigma=\operatorname{Re} s>1$, in view of the classical Riemann-von Mangoldt formula

$$
N(T)=\frac{T}{2 \pi} \log \frac{T}{2 \pi}-\frac{T}{2 \pi}+O(\log T)
$$

for the zero counting function $N(T):=\sum_{0<\gamma \leqslant T} 1$ (see [Iv03, p. 17] or [Ti86, p. 214]). The condition $\gamma>0$ in (1.1) is natural, since the property $\overline{\zeta(s)}=\zeta(\bar{s})$ ensures that $\beta-i \gamma$ is a zero of $\zeta(s)$ if $\beta+i \gamma$ is a zero.

The function $G(s)$ is mentioned in the work of Delsarte [Del66] and, in a perfunctory way, in the works of Chakravarty [Chak70, Chak71]. A related zeta function, namely

$$
\sum_{\gamma>0} \gamma^{-s} \sin (\alpha \gamma) \quad(\alpha>0)
$$

was studied by Fujii [Fu83], but its properties are different from those of $G(s)$, and we shall not consider it here. Both Chakravarty and Delsarte (as well as Fujii) assume the Riemann hypothesis (that all complex zeros of $\zeta(s)$ satisfy $\operatorname{Re} s=1 / 2$ ) when dealing with $G(s)$. Delsarte [Del66, p. 430] obtains its analytic continuation to $\mathbb{C}$ by employing Poisson summation and a sort of a modular relation.

We thank episciences.org for providing open access hosting of the electronic journal Hardy-Ramanujan Journal

Saksman's research was supported in part by the Lars Onsager Professorship at NTNU and in part by the Finnish Academy CoE "Analysis and Dynamics". The research of Bondarenko and Seip was supported in part by Grant 227768 of the Research Council of Norway. 
We discuss analytic continuation of Delsarte's function $G(s)$ in Section 2. We give a different proof of Delsarte's result on the analytic continuation under the Riemann hypothesis. Moreover, we note that meromorphic extension to a larger half-plane $\sigma>-1-\varepsilon$ would have strong consequences related to the density hypothesis. In Section 3., we provide an explicit Laurent expansion of $G(s)$ at $s=1$. Section 4 . is devoted to estimating the second moment $\int_{1}^{T}\left|G\left(\frac{1}{2}+i t\right)\right|^{2} \mathrm{~d} t$. There the growth of the second moment is connected explicitly to the fluctuations of the function $S(t)$ (see (2.5) below), and we provide lower and upper bounds for the growth. Finally, in Section 5. we observe how the connection of $G(s)$ to the "super zeta functions" studied in the monograph [Vor10] $]^{1}$ leads to yet another approach to Delsarte's result.

\section{Analytic continuation of $G(s)$}

The second author introduced and studied $G(s)$ in [Iv01]. Starting from the classical formula

$$
N(T)=\frac{T}{2 \pi} \log \frac{T}{2 \pi}-\frac{T}{2 \pi}+\frac{7}{8}+S(T)+f(T),
$$

it was shown that, for $\sigma>0$ and a suitable constant $C_{1}$,

$$
G(s)=\frac{1}{2 \pi(s-1)^{2}}-\frac{\log (2 \pi)}{2 \pi(s-1)}+C_{1}+s \int_{1}^{\infty}(S(x)+f(x)) x^{-s-1} \mathrm{~d} x .
$$

Here, if $T$ is not an ordinate of a zero,

$$
S(T)=\frac{1}{\pi} \arg \zeta\left(\frac{1}{2}+i T\right)=\frac{1}{\pi} \operatorname{Im}\left\{\log \zeta\left(\frac{1}{2}+i T\right)\right\} \ll \log T,
$$

where the argument of $\zeta\left(\frac{1}{2}+i T\right)$ is obtained by continuous variation of the argument of $\zeta(s)$ along the straight lines joining the points $2,2+i T, \frac{1}{2}+i T$, starting with the value 0 at $s=2$. If $T$ is an ordinate of a zero, then $S(T)=S(T+0)$. The function $f(T)$ is smooth and admits an asymptotic expansion in terms of negative odd powers of $T$, namely for any given integer $N \geqslant 1$ one has

$$
f(T)=\sum_{j=1}^{N} \frac{a_{j}}{T^{2 j-1}}+O_{N}\left(\frac{1}{T^{2 N+1}}\right),
$$

where the $a_{j}$ are explicit constants, and $O_{N}$ means that the implied $O$-constant depends only on $N$. From (2.4)-(2.6) one obtains the analytic continuation of $G(s)$ for $\sigma>0$.

Further analytic continuation will follow by integrating the integral in (2.4) by parts. This will give, for $\sigma>-1$ and a suitable constant $C_{1}$,

$$
\begin{aligned}
G(s)= & \frac{1}{2 \pi(s-1)^{2}}-\frac{\log 2 \pi}{2 \pi(s-1)}+C_{1}+s \int_{1}^{\infty} f(x) x^{-s-1} \mathrm{~d} x \\
& +s(s+1) \int_{1}^{\infty} \int_{1}^{x} S(u) \mathrm{d} u \cdot x^{-s-2} \mathrm{~d} x
\end{aligned}
$$

since we have the (unconditional) bound (see [Ti86, pp. 221-222])

$$
\int_{0}^{T} S(t) \mathrm{d} t=O(\log T)
$$

\footnotetext{
${ }^{1}$ The study of such zeta functions goes back to Mellin [Mel17] in 1916 and seems not to be widely known.
} 
To study the analytic continuation of $G(s)$ for $\sigma=\operatorname{Re} s \leqslant-1$ we need some notation. Following [Fu02], we define

$$
\tilde{S}_{0}(T):=S(T) \quad(T \neq \gamma)
$$

and for $m \geqslant 1$,

$$
\tilde{S}_{m}(T):=\int_{0}^{T} \tilde{S}_{m-1}(t) \mathrm{d} t+C_{m}
$$

where $C_{2 k}:=(-1)^{k-1} /\left((2 k) ! 4^{k}\right)$ and

$$
C_{2 k-1}:=\frac{(-1)^{k-1}}{\pi} \int_{\frac{1}{2}}^{\infty} \int_{\sigma_{1}}^{\infty} \cdots \int_{\sigma_{2 k-2}}^{\infty} \log \left|\zeta\left(\sigma_{2 k-1}\right)\right| \mathrm{d} \sigma_{2 k-1} \cdots \mathrm{d} \sigma_{1}
$$

for every positive integer $k$. When $T=\gamma$, we put

$$
\tilde{S}_{m}(T):=\frac{1}{2}\left(\tilde{S}_{m}(T+0)+\tilde{S}_{m}(T-0)\right) .
$$

On the Riemann hypothesis, it is known that (see [Ti86, pp. 350-354])

$$
\tilde{S}_{m}(T) \ll_{m} \frac{\log T}{(\log \log T)^{m+1}} \quad(m \geqslant 1) .
$$

Here $\ll_{m}$ means that the constant implied by the $\ll$-symbol depends only on $m$. Note that

$$
\int_{1}^{\infty} x^{-2 j+1-s-1} \mathrm{~d} x=\frac{1}{2 j+s-1} \quad(\sigma>1-2 j, j \in \mathbb{N}) .
$$

Thus using (2.6) and (2.11), we see that

$$
s \int_{1}^{\infty} f(x) x^{-s-1} \mathrm{~d} x
$$

in (2.7) represents a meromorphic function in $\mathbb{C}$, which has simple poles at $j=-1,-3, \ldots$. Hence repeated integrations by parts of the last term in (2.4) show, on using (2.9), that under the Riemann hypothesis the function $G(s)$ admits meromorphic continuation to $\mathbb{C}$. Its only poles are: $s=1$ (order two) and $s=-1,-3, \ldots$ (order one).

We next look at what are the consequences if we do not assume the Riemann hypothesis to hold, but instead assume that $G(s)$ admits a meromorphic continuation across the line $\sigma=-1$. The integration by parts argument used in the preceding discussion implies in particular that then the function

$$
H_{1}(s):=\int_{1}^{\infty} \tilde{S}_{2}(x) x^{-s-3} \mathrm{~d} x
$$

continues meromorphically across the same line. By [Fu02, Theorem 3], we have

$$
\tilde{S}_{2}(T)=\sum_{\beta>1 / 2,0<\gamma \leqslant T}(\beta-1 / 2)^{2}+O(\log T)=A(T)+O(\log T),
$$

where we have set $A(T):=\sum_{\beta>1 / 2,0<\gamma \leqslant T}(\beta-1 / 2)^{2}$. We therefore obtain that also the function

$$
H(s):=\int_{1}^{\infty} A(x) x^{-s-3} \mathrm{~d} x
$$

extends meromorphically across $\sigma=-1$. 
Now let as usual $N(\sigma, T)$ be the number of zeros $\beta+i \gamma$ with $\frac{1}{2} \leqslant \sigma<\beta<1$ and $0<\gamma \leqslant T$. We may then write

$$
A(x)=2 \int_{1 / 2}^{1}\left(\sigma-\frac{1}{2}\right) N(\sigma, x) \mathrm{d} \sigma .
$$

Namely the right-hand side above equals

$$
\begin{aligned}
2 \int_{1 / 2}^{1}\left(\sigma-\frac{1}{2}\right) \sum_{\gamma \leqslant x, \beta \geqslant \sigma \geqslant \frac{1}{2}} 1 \mathrm{~d} \sigma & =2 \sum_{\gamma \leqslant x} \int_{\frac{1}{2}}^{\beta}\left(\sigma-\frac{1}{2}\right) \mathrm{d} \sigma \\
& =\sum_{\gamma \leqslant x, \beta \geqslant \frac{1}{2}}\left(\beta-\frac{1}{2}\right)^{2}=A(x) .
\end{aligned}
$$

By Selberg's uniform density estimate [Ti86, Theorem $9.19(\mathrm{C})]$,

$$
A(x) \ll x \log x \int_{0}^{1 / 2} y x^{-y / 4} \mathrm{~d} y \ll \frac{x}{\log x} .
$$

Now

$$
\int_{e}^{\infty} \frac{x^{-\varepsilon-1}}{\log x} \mathrm{~d} x=\int_{1}^{\infty} \frac{e^{-\varepsilon u}}{u} \mathrm{~d} u \leqslant \int_{1}^{1 / \varepsilon} \frac{\mathrm{d} u}{u}+\varepsilon \int_{0}^{\infty} e^{-\varepsilon u} \mathrm{~d} u \leqslant \log \frac{1}{\varepsilon}+1 .
$$

Hence $H(s)$ cannot have a pole at $s=-1$, and by our assumption it thus has to be analytic at $s=-1$. Then since $A$ is non-negative and increasing, a classical theorem of Landau [Ten95, Theorem 6, Chapter II.1] implies that there is $\delta>0$ such that

$$
A(x) \ll x^{1-\delta} .
$$

It follows from the definition of $A(x)$ and the fact that $\sigma \mapsto N(\sigma, x)$ is a decreasing function that for $\sigma>1 / 2$

$$
N(\sigma, T) \ll(\sigma-1 / 2)^{-2} T^{1-\delta},
$$

with the implicit constant depending only on $\delta$. For $\sigma$ close to $1 / 2$, this assertion is stronger than the density hypothesis that $N(\sigma, T) \ll_{\varepsilon} T^{2-2 \sigma+\varepsilon}(1 / 2 \leqslant \sigma \leqslant 1)$.

We summarize the preceding discussion as follows:

Theorem 2.1. Unconditionally, $G(s)$ has meromorphic continuation at least to $\sigma>-1$; it has only one pole in this half-plane, located at $s=1$ and of order two. If the Riemann hypothesis is true, then $G(s)$ extends meromorphically to $\mathbb{C}$ with additional poles that are all simple and located at $s=-1,-3, \ldots$. If $G(s)$ continues meromorphically across $\sigma=-1$, then the strong implication (2.14) holds for the density of the zeros off the critical line.

Remark 2.2. As noted in Section 1., the meromorphic continuation of $G(s)$ to $\mathbb{C}$ on the Riemann hypothesis was already obtained by Delsarte [Del66], but the above proof is simpler (see also Section 5. below). The last assertion of Theorem 2.1 indicates that establishing meromorphic continuation across the line $\sigma=-1$ is likely to be very difficult.

Notice that we may have meromorphic extension to the whole complex plane even if the Riemann hypothesis fails. This will for instance be the case if there are finitely many nontrivial zeros $\rho=\beta+i \gamma$ with $\beta>1 / 2$. To see this, we write (2.13) in the form $\tilde{S}_{2}(T)=A(T)+I_{2}(T)$. Plugging this into (2.12), we see that the term $A(x)$ gives rise to an additional simple pole at -2 , while the term $I_{2}(x)$, by repeated integration by parts and use of [Fu02, Theorem 1], yields an entire function. 
An elaboration of this observation, along the lines of our discussion of possible meromorphic continuation across $\sigma=-1$, shows that the distinctive feature of the meromorphic continuation obtained on the Riemann hypothesis is that $G(s)$ will be analytic on the segment $[-2,-1)$. This leads to the following statement.

Theorem 2.3. The Riemann hypothesis holds if and only if $G(s)$ has meromorphic continuation to the half-plane $\sigma \geqslant-2$ with only two poles that are located at respectively 1 and -1 .

\section{The Laurent expansion of $G(s)$ at $s=1$}

From (2.4) one can obtain the (unconditional) Laurent expansion of $G(s)$ at its pole $s=1$. Namely, denoting $F(x):=S(x)+f(x)$ for brevity, we have for $|s-1|<1$,

$$
\int_{1}^{\infty} F(x) x^{-s-1} \mathrm{~d} x=\int_{1}^{\infty} F(x) e^{-(s-1) \log x} x^{-2} \mathrm{~d} x=\sum_{j=0}^{\infty} c_{j}(s-1)^{j},
$$

say, with

$$
c_{j}:=\frac{(-1)^{j}}{j !} \int_{1}^{\infty} F(x) \log ^{j} x \cdot x^{-2} \mathrm{~d} x \quad(j=0,1, \ldots) .
$$

Here we could interchange integration and summation in view of absolute convergence; this follows from the bound $F(x) \ll \log x$ which holds because $S(x) \ll \log x$. Writing the last expression in (2.4) as

$$
(s-1) \int_{1}^{\infty} F(x) x^{-s-1} \mathrm{~d} x+\int_{1}^{\infty} F(x) x^{-s-1} \mathrm{~d} x
$$

and using (3.15), we obtain

Theorem 3.1. The Laurent expansion of $G(s)$ at $s=1$ has the form

$$
G(s)=\frac{1}{2 \pi(s-1)^{2}}-\frac{\log 2 \pi}{2 \pi(s-1)}+\sum_{j=0}^{\infty} b_{j}(s-1)^{j},
$$

where

$$
b_{0}=C_{1}+c_{0}, \quad b_{j}=c_{j}+c_{j-1} \quad(j=1,2, \ldots),
$$

and the $c_{j}$ are given by (3.16).

\section{Estimates for the second moment}

We will now provide upper and lower bounds for the second moment

$$
\int_{1}^{T}\left|G\left(\frac{1}{2}+i t\right)\right|^{2} \mathrm{~d} t
$$

thus continuing the investigations started in [Iv01, Section 2]. To this end, we follow [Iv01] and write

$$
G(s)=\sum_{\gamma \leqslant X} \gamma^{-s}+R(s),
$$

where

$$
R(s)=\int_{X}^{\infty} \frac{x^{-s}}{2 \pi} \log \frac{x}{2 \pi} \mathrm{d} x+\int_{X}^{\infty} x^{-s} \mathrm{~d}(S(x)+f(x)) .
$$


Integrating by parts, we get

$$
R(s)=\frac{X^{1-s}}{2 \pi(s-1)} \log \frac{X}{2 \pi}+\frac{X^{1-s}}{2 \pi(s-1)^{2}}-X^{-s}(S(X)+f(X))+s \int_{X}^{\infty} x^{-s-1}(S(x)+f(x)) \mathrm{d} x,
$$

which to begin with is valid for $\sigma>1$, but which continues meromorphically for all $\sigma>0$. For $s=1 / 2+i t$, the integral on the right-hand side of (4.17) may be written as $\widehat{g}(t / 2 \pi)$, where $g(\xi):=$ $e^{-\xi / 2}\left(S\left(e^{\xi}\right)+f\left(e^{\xi}\right)\right) \chi_{[X, \infty)}\left(e^{\xi}\right)$. Hence elementary estimates and Parseval's identity together with Selberg's bound $\int_{T}^{2 T} S^{2}(t) \mathrm{d} t \ll T \log \log T$ yield

$$
\int_{1}^{T}\left|R\left(\frac{1}{2}+i t\right)\right|^{2} \mathrm{~d} t \ll X \log ^{2} X+\left(T^{2} / X\right) \log \log X .
$$

Choosing $X=T \sqrt{\log \log T} / \log T$ we get

$$
\int_{1}^{T}\left|R\left(\frac{1}{2}+i t\right)\right|^{2} \mathrm{~d} t \ll T \log T \sqrt{\log \log T}
$$

As we will see in (4.21) below, $\int_{1}^{T}\left|\sum_{\gamma \leqslant X} \gamma^{-1 / 2-i t}\right|^{2} \mathrm{~d} t \gg T \log ^{2} T$ when $X \geqslant T^{b}$ for some positive number $b$. Hence the second moment of the partial sum $\sum_{\gamma \leq X} \gamma^{-1 / 2-i t}$ yields the dominant term which needs to be studied in more detail.

Let $\psi$ be a symmetric function in $C(\mathbb{R})$ satisfying $^{2}$

$$
0 \leqslant \psi(x) \leqslant \chi_{[-1,1]}(x) \text { and } 0<c_{1} \leqslant\left(1+4 \pi^{2}|\xi|\right)^{2} \widehat{\psi}(\xi) \leqslant c_{2}
$$

for all $\xi$ in $\mathbb{R}$. Let $A$ be an arbitrary subset of $\{\gamma: 0<\gamma \leqslant T\}$. We find that

$$
\begin{aligned}
\int_{-T}^{T}\left|\sum_{\gamma \in A} \gamma^{-1 / 2-i t}\right|^{2} \mathrm{~d} t & \geqslant \int_{\mathbb{R}}\left|\sum_{\gamma \in A} \gamma^{-1 / 2-i t}\right|^{2} \psi(t / T) \mathrm{d} t=T \sum_{\gamma, \gamma^{\prime} \in A}\left(\gamma \gamma^{\prime}\right)^{-1 / 2} \widehat{\psi}\left(T \log \left(\gamma / \gamma^{\prime}\right) / 2 \pi\right) \\
& \asymp T \sum_{\gamma, \gamma^{\prime} \in A}\left(\gamma \gamma^{\prime}\right)^{-1 / 2} \frac{1}{1+T^{2}\left(\log \left(\gamma^{\prime} / \gamma\right)\right)^{2}} .
\end{aligned}
$$

Fix an arbitrary constant $a>0$ and replace $\psi(x)$ by $\widehat{\psi}(x /(2 \pi a))$ in the above computation. We obviously obtain an upper estimate up to a constant, but as $\psi(a \cdot) \ll \widehat{\psi}(\cdot /(2 \pi)) \asymp \widehat{\psi}(a \cdot /(2 \pi))$, we infer that

$$
\begin{aligned}
\int_{-T}^{T}\left|\sum_{\gamma \in A} \gamma^{-1 / 2-i t}\right|^{2} \mathrm{~d} t & \asymp T \sum_{\gamma, \gamma^{\prime} \in A}\left(\gamma \gamma^{\prime}\right)^{-1 / 2} \frac{1}{1+T^{2}\left(\log \left(\gamma^{\prime} / \gamma\right)\right)^{2}} \\
& \asymp T \sum_{\gamma, \gamma^{\prime} \in A}\left(\gamma \gamma^{\prime}\right)^{-1 / 2} \psi\left(T a \log \left(\gamma^{\prime} / \gamma\right)\right) \\
& \asymp T \sum_{\gamma \in A} \gamma^{-1} \#\left\{\gamma^{\prime} \in(0, T) \cap A:\left|\gamma^{\prime}-\gamma\right| \leqslant \gamma /(a T)\right\} .
\end{aligned}
$$

As the parameter $a$ is arbitrary, we see that there is some flexibility in choosing the size of the "window" in (4.23) yielding the pairs of ordinates that contribute significantly to the second moment.

By considering only the diagonal $\gamma=\gamma^{\prime}$ in (4.19) and using the known average density of the ordinates, we obtain

$$
\int_{-T}^{T}\left|\sum_{0<\gamma \leqslant X} \gamma^{-1 / 2-i t}\right|^{2} \mathrm{~d} t \gg T \sum_{0<\gamma \leq T^{b}} \gamma^{-1} \gg T \log ^{2} T
$$

${ }^{2}$ We may for example choose $\psi(x)=(\max (1-|x|), 0)^{2}$. 
whenever $X \geqslant T^{b}$ for some exponent $b$ in $(0,1)$.

Our next goal is to express (4.19) in terms of either of the functions $N(t)$ and $S(t)$ in the special case when $A=\{\gamma: 0<\gamma \leqslant X\}$. We start with the basic formula involving $N(t)$.

Lemma 4.1. Suppose that $100 \leqslant X \leqslant T$. Then

$$
\int_{1}^{T}\left|\sum_{0<\gamma \leqslant X} \gamma^{-1 / 2-i t}\right|^{2} \mathrm{~d} t \asymp \int_{1}^{X}\left(\frac{N(t+t / T)-N(t)}{t / T}\right)^{2} \mathrm{~d} t .
$$

Proof. We start from (4.19) in the form

$$
\int_{-T}^{T}\left|\sum_{0<\gamma \leqslant X} \gamma^{-1 / 2-i t}\right|^{2} \mathrm{~d} t \asymp T \sum_{1<\gamma \leq X} \gamma^{-1} \#\left\{\gamma^{\prime}:\left|\gamma^{\prime}-\gamma\right| \leqslant \gamma /(a T)\right\} .
$$

Here we discarded the condition that $\gamma^{\prime} \leqslant X$ in the sum on the right-hand side. The right-hand side has thus been increased by a term of size $O\left((\log X)^{2} T / X\right)$ which is admissible because the left-hand side is $\gg T \log ^{2} X$. We have also taken into account that $\gamma>0$ implies $\gamma>1$.

Set $k_{0}:=\lfloor T \log X\rfloor$ and $b_{k}:=X^{k / k_{0}}$. We divide $(1, X]$ into intervals $I_{k}=\left(b_{k-1}, b_{k}\right]$ for $k=$ $1, \ldots, k_{0}+1$; it will be convenient to agree that $I_{0}=\emptyset$. For any real interval $I$ let $n(I)$ denote the number of zeta zeros with ordinates in $I$. Then for an arbitrary $\gamma$ in $I_{k}$ we obtain

$$
\#\left\{\gamma^{\prime} \in(0, T):\left|\gamma^{\prime}-\gamma\right|<\gamma / T\right\} \leqslant n\left(I_{k-1}\right)+n\left(I_{k}\right)+n\left(I_{k+1}\right) .
$$

It follows that

$$
\begin{aligned}
\sum_{\gamma \in\left(b_{k-1}, b_{k}\right]} \gamma^{-1} \#\left\{\gamma^{\prime} \in(0, T):\left|\gamma^{\prime}-\gamma\right| \leqslant \gamma / T\right\} & \leqslant b_{k-1}^{-1} n\left(I_{k}\right)\left(n\left(I_{k-1}\right)+n\left(I_{k}\right)+n\left(I_{k+1}\right)\right) \\
& \ll b_{k-1}^{-1} n\left(I_{k-1}\right)^{2}+b_{k}^{-1} n\left(I_{k}\right)^{2}+b_{k+1}^{-1} n\left(I_{k+1}\right)^{2} .
\end{aligned}
$$

On the other hand, we clearly have

$$
\sum_{\gamma \in\left(b_{k-1}, b_{k}\right]} \gamma^{-1} \#\left\{\gamma^{\prime} \in(0, T):\left|\gamma^{\prime}-\gamma\right| \leqslant 2 \gamma / T\right\} \geqslant b_{k}^{-1} n\left(I_{k}\right)^{2} .
$$

Summing both the upper and lower estimates over $k$ and using (4.23), we get

$$
\int_{-T}^{T}\left|\sum_{0<\gamma \leqslant X} \gamma^{-1 / 2-i t}\right|^{2} \mathrm{~d} t \asymp T \sum_{k} b_{k}^{-1} n\left(I_{k}\right)^{2} .
$$

We may neglect the first interval and consider $I_{k}$ with $k \geqslant 2$. Divide each $I_{k}$ into three equally long subintervals. One of these three intervals contains at least $n\left(I_{k}\right) / 3$ ordinates, and there is then a subinterval $I_{k}^{\prime}$ of $I_{k} \cup I_{k-1}$ with $\left|I_{k}^{\prime}\right| \geq\left|I_{k}\right| / 3$ such that $N(t+t / T)-N(t) \geq n\left(I_{k}\right) / 3$ for every $t$ in $I_{k}^{\prime}$. Since $\left|I_{k}\right| \sim b_{k} / T \asymp t / T$ for $t$ in $2 I_{k}$ we thus obtain

$$
\begin{aligned}
T b_{k}^{-1} n\left(I_{k}\right)^{2} & \left.\ll T b_{k}^{-1}\left|I_{k}\right|^{-1} \int_{I_{k-1} \cup I_{k}}(N(t+t / T)-N(t))^{2}\right) \mathrm{d} t \\
& \left.\ll \int_{I_{k-1} \cup I_{k}}(N(t+t / T)-N(t))^{2}\right) \frac{\mathrm{d} t T^{2}}{t^{2}} \ll T b_{k}^{-1}\left(n\left(I_{k-1}\right)+n\left(I_{k}\right)+n\left(I_{k+1}\right)\right)^{2},
\end{aligned}
$$

where the last inequality follows on noting that $N(t+t / T)-N(t) \leqslant n\left(I_{k}\right)+n\left(I_{k+1}\right)$ for interior points $t$ in $I_{k}$. Summing over $k$ and using (4.24), we finally arrive at (4.22). 
We may alternatively replace $N(t)$ by $S(t)$ in $(4.22)$ :

$$
\int_{1}^{T}\left|\sum_{0<\gamma \leqslant X} \gamma^{-1 / 2-i t}\right|^{2} \mathrm{~d} t \asymp \int_{1}^{X}\left(\frac{S(t+t / T)-S(t)}{t / T}\right)^{2} \mathrm{~d} t .
$$

Indeed, by (2.3), (4.22) implies that

$$
\int_{1}^{T}\left|\sum_{0<\gamma \leqslant X} \gamma^{-1 / 2-i t}\right|^{2} \mathrm{~d} t \ll \int_{1}^{X}\left(\frac{S(t+t / T)-S(t)}{t / T}\right)^{2} \mathrm{~d} t+X \log ^{2} X .
$$

Since the left-hand side is $\gg T \log ^{2} X$, we get that the second term on the right-hand side can be removed. Since (2.3) implies that

$$
N(t+t / T)-N(t) \geqslant S(t+t / T)-S(t)
$$

it is also clear that (4.22) trivially implies

$$
\int_{1}^{T}\left|\sum_{0<\gamma \leqslant X} \gamma^{-1 / 2-i t}\right|^{2} \mathrm{~d} t \gg \int_{1}^{X}\left(\frac{S(t+t / T)-S(t)}{t / T}\right)^{2} \mathrm{~d} t .
$$

The above lemma, as expressed by (4.25) shows that the asymptotics that we are interested in, depends crucially on the oscillations of $S(t)$ in rather small intervals. To gain more insight into this asymptotics, we will next take a closer look at the size of the dyadic parts

$$
D(x):=\int_{1}^{T}\left|\sum_{x<\gamma \leqslant 2 x} \gamma^{-1 / 2-i t}\right|^{2} \mathrm{~d} t .
$$

Proposition 4.2. (i) Assume that $T / \log T \leq x \leq T$. Then

$$
x \log ^{2} T \ll D(x) \ll T \log ^{2} T .
$$

(ii) Assume that $100 \leqslant x<T / \log T$. Then

$$
T \log x \ll D(x) \ll T \log x \sqrt{\log \log x} .
$$

Proof of part (i) of Proposition 4.2. We may assume that $T \gg 1$ is an integer. Divide the interval $(x, 2 x]$ into $T$ subintervals $I_{j}:=(x+(j-1) x / T, x+j x / T]$ for $1 \leqslant j \leq T$. By (4.19), arguing as in the proof of (4.24), we then get

$$
D(x) \asymp \frac{T}{x} \sum_{x \leqslant \gamma<2 x}\left|\left\{\gamma^{\prime}:\left|\gamma-\gamma^{\prime}\right| \leqslant \frac{x}{T}\right\}\right| \asymp \frac{T}{x} \sum_{j=1}^{T} n\left(I_{j}\right)^{2} .
$$

Since we know that $\sum_{j=1}^{T} n\left(I_{j}\right)=n([x, 2 x)) \asymp x \log x$, an application of the Cauchy-Schwarz inequality to (4.26) yields immediately the lower bound $D(x) \geqslant x \log ^{2} x \asymp x \log ^{2} T$. To obtain the upper bound, we divide the ordinates in $[x, 2 x)$ into $O(\log T) 1$-separated sets, and apply the MontgomeryVaughan inequality [MoVa74, Corollary 2] to each of the corresponding sums.

In the proof of the upper bound of part (ii), we will use the following lemma. 
Lemma 4.3. There is a universal constant $c>0$ such that if $n(I) \geqslant|I| \log x$ for a subinterval $I=[a, b]$ of $[x, 2 x+x / T]$, then

$$
|\{t \in 2 I:|S(t)| \geqslant n(I) / 8\}| \geqslant c|I| .
$$

Proof. By the Riemann-von Mangoldt formula, the assumption $n(I) \geqslant|I| \log x$ implies that

$$
S(b)-S(a) \geqslant \frac{1}{2}|I| \log x
$$

This implies that if $S(b) \leqslant \frac{1}{4}|I| \log x$, then $S(a) \leq-\frac{1}{4}|I| \log x$ in which case may choose a positive constant $c$ independent of $I$ such that the desired bound $|S(t)| \geqslant n(I) / 8$ holds on the interval $[a-c|I| \log x, a]$. On the other hand, if $S(b)>\frac{1}{4}|I| \log x$, then $|S(t)| \geqslant n(I) / 8$ holds on the interval $[b, b+c|I| \log x]$.

Proof of part (ii) of Proposition 4.2. We divide the interval $(x, 2 x]$ into the same subintervals $I_{j}$ as in the preceding case. The lower bound of this part is again proved by the Cauchy-Schwarz inequality. Indeed, observing that the number of non-zero summands in the sum over $j$ is at most of order $x \log x$, we find that

$$
D(x) \asymp \frac{T}{x^{2} \log x} x \log x \sum_{j=1}^{T} n\left(I_{j}\right)^{2} \geqslant \frac{T}{x^{2} \log x}(x \log x)^{2}=T \log x .
$$

We turn to the proof of the upper bound. To begin with, note that since $x \leqslant T / \log T$, we have $\left|I_{j}\right| \log x \leq 1$. This means that $I_{j}$ satisfies the condition of Lemma 4.3 whenever $n\left(I_{j}\right) \geqslant 1$. This fact allows us to make the following construction. Let $j_{1}$ be the smallest $j$ such that $n\left(I_{j}\right) \geqslant 1$, and let $k_{1}$ be the largest $k$ such that

$$
n\left(\bigcup_{m=0}^{k-1} I_{j_{1}+m}\right) \geqslant \frac{k x}{T} \log x
$$

Now choose $j_{\ell}$ and $k_{\ell}$ inductively such that $j_{\ell}$ is the smallest $j$ such that $j \geqslant j_{\ell-1}+k_{\ell-1}$ and $n\left(I_{j}\right) \geqslant 1$, while $k_{\ell}$ is the largest $k$ such that

$$
n\left(\bigcup_{m=0}^{k-1} I_{j_{\ell}+m}\right) \geqslant \frac{k x}{T} \log x
$$

We terminate this iteration when $j_{\ell}+k_{\ell} \geqslant 2 T$. We set

$$
J_{\ell}:=\bigcup_{m=0}^{k_{\ell}-1} I_{j_{\ell}+m}
$$

and notice, by the maximality of $J_{\ell}$, that

$$
n\left(J_{\ell}\right)=(1+o(1))\left|J_{\ell}\right| \log x
$$

Plainly by the construction, since $x \leqslant T / \log T$, all the ordinates $\gamma$ in $[x, 2 x]$ will be covered by the intervals $J_{\ell}$, and we thus have

$$
\sum_{j} n\left(I_{j}\right)^{2} \leqslant \sum_{\ell} n\left(J_{\ell}\right)^{2}
$$


Now, by a standard covering argument (see e.g. [Gar07, Proof of Lemma 4.4]), we can find a subcollection $\left\{2 J^{\prime}\right\}$ of the intervals $2 J_{\ell}^{3}$ such that no point in $[x, 2 x]$ belongs to more than two intervals $2 J^{\prime}$ and such that the union of $\left\{2 J^{\prime}\right\}$ covers all the intervals $J_{\ell}$. In particular, we have

$$
\sum_{\ell}\left|J_{\ell}\right|^{2} \ll \sum_{J^{\prime}}\left|J^{\prime}\right|^{2}
$$

Combining this with Lemma 4.3 and (4.27), we get

$$
\begin{aligned}
\sum_{\ell} n\left(J_{\ell}\right)^{2} & \ll \log x \sum_{\ell}(\log x)\left|J_{\ell}\right| \cdot\left|J_{\ell}\right| \ll \log x \sum_{J^{\prime}}(\log x)\left|J^{\prime}\right| \cdot\left|J^{\prime}\right| \\
& \ll \log x \int_{x}^{2 x}|S(t)| \mathrm{d} t \ll x \log x \sqrt{\log \log x},
\end{aligned}
$$

where in the last step we used the Cauchy-Schwarz inequality and Selberg's mean value theorem ${ }^{4}$ for $S(t)$. Recalling (4.28) and (4.26), we obtain the upper bound of part (ii).

As an immediate consequence of the preceding analysis, we now obtain a mean square estimate ${ }^{5}$ for the function $G(s)$ on the critical line:

Theorem 4.4. $\quad T \log ^{2} T \ll \int_{1}^{T}\left|G\left(\frac{1}{2}+i t\right)\right|^{2} \mathrm{~d} t \ll T \log ^{2} T \sqrt{\log \log T}$.

Proof. We choose the length $X$ of the partial sum $\sum_{0<\gamma \leqslant X} \gamma^{-s}$ to be $T \sqrt{\log \log T} / \log T$ so that the contribution from the remainder term is given by (4.18). By (4.24) (or directly by (4.19)) there is quasi-orthogonality between the dyadic parts of the sum (i.e., dyadic parts that are not adjacent are orthogonal with respect to a weighted norm, where the size of the weight is approximately $\left(1+|t / T|^{2}\right)$ ). We apply part (i) of Proposition 4.2 to suitable dyadic subintervals of $[T / \log T, T \sqrt{\log \log T} / \log T]$ and part (ii) to subintervals of $[1, T / \log T]$. Summing over all these dyadic intervals, we obtain the desired upper bound. The lower bound is a consequence of (4.21) and (4.18).

We expect, partly in view of the very regular behavior of the ordinates predicted by the random matrix analogy, that the true order for dyadic summands is akin to something like

$$
D(x) \asymp \begin{cases}x \log ^{2} T & \text { for } T / \log T \leqslant x \leqslant T, \\ T \log x & \text { for } x \leqslant T / \log T .\end{cases}
$$

We note that this prediction corresponds to the lower bounds in Proposition 4.2. This leads us to the following

Conjecture 4.5. $\quad \int_{1}^{T}\left|G\left(\frac{1}{2}+i t\right)\right|^{2} \mathrm{~d} t \asymp T \log ^{2} T$.

Remark 4.6. On the Riemann hypothesis, we may split the sequence of ordinates $\gamma$ in the range $T / \log T \leqslant \gamma \leqslant T$ in $O(\log T / \log \log T)$ sequences that are all separated with separation constant $\geqslant 1 / \log \log T$. Using again the Montgomery-Vaughan inequality, we then obtain the conjectured bound for the sum over $\gamma$ in this restricted range, namely

$$
\int_{1}^{T}\left|\sum_{T / \log T \leqslant \gamma \leqslant T} \gamma^{-1 / 2-i t}\right|^{2} \mathrm{~d} t \ll T \log ^{2} T .
$$

\footnotetext{
${ }^{3}$ If $I \subset \mathbb{R}$ is an interval, $2 I$ stands for the interval with same center and twice the length.

${ }^{4}$ Alternatively, we could appeal to Ghosh's asymptotic formula for the first moment of $S(t)$ [Gho81].

${ }^{5}$ The upper bound $\int_{1}^{T}\left|G\left(\frac{1}{2}+i t\right)\right|^{2} \mathrm{~d} t \ll T \log ^{2} T$ was stated in [Iv01] but with an incomplete proof.
} 


\section{On super zeta functions}

In 1917 Mellin [Mel17] considered a class of zeta functions that are defined by using the Riemann zeta zeros as the building block of new Dirichlet series. For example, he established meromorphic continuation to the whole complex plane for a class of such functions including the function

$$
Z(s):=\sum_{\rho} \rho^{-s}
$$

where the sum is taken over all nontrivial zeros of the Riemann zeta function. Voros has devoted a monograph [Vor10] on generalisations of such functions, for which he coins the name super zeta functions $^{6}$.

For the convenience of the reader, we now sketch a proof of the existence of a meromorphic extension of the function

$$
M_{\alpha}(s):=\sum_{\rho}(\alpha-\rho)^{-s}
$$

to the whole complex plane. This function is initially well-defined in the half-plane $\sigma>1$, as one sets $(\alpha-w)^{-s}:=e^{-s \log (\alpha-w)}$ for $w$ in $\mathbb{C} \backslash[\alpha,+\infty)$, using the convention that $\log (\alpha-w)$ is real-valued for real $w<\alpha$. We will will consider only $M_{\alpha}$ with $\alpha>-2$, although we could consider the more general case. However, our main interest lies in the case $\alpha=1 / 2$, which under the Riemann hypothesis yields another approach to Delsarte's result. We refer to [Vor10, Section 7] which contains a detailed discussion of the analytic continuation, and also note that [Vor10, Appendix D] provides an English translation of Mellin's work [Mel17] which was originally written in German.

Clearly (5.30) defines a function analytic in the half-plane $\sigma>1$. Set $c:=\min (1, \alpha)$ and let $\eta_{\alpha}$ be the "Hankel contour" going from $+\infty$ below the real line to $c-i \varepsilon$, where it follows the semicircle of radius $\varepsilon<2+\alpha$ centred at $c$ to the left of $c$ to $c+i \varepsilon$, and then going back to $+\infty$. Then the integral

$$
E_{\alpha}(s):=\frac{1}{2 \pi i} \int_{\eta_{\alpha}} \frac{\zeta^{\prime}(w)}{\zeta(w)}(\alpha-w)^{-s} \mathrm{~d} w
$$

defines an entire function $E_{\alpha}$, by the rapid decay of $\zeta^{\prime}(w) / \zeta(w)$. For $s>1$, the contour can be moved into the left half-plane and towards $-\infty$ in the usual way and therefore

$$
\zeta_{\alpha}(s)=E_{\alpha}(s)-\sum_{n=0}^{\infty}(\alpha+2+2 n)^{-s}=E_{\alpha}(s)-2^{-s} \zeta(s, \alpha / 2+1),
$$

where as usual $\zeta(s, q)$ is the Hurwitz zeta function. The meromorphic continuation follows since the Hurwitz zeta function is known to be a meromorphic function in $\mathbb{C}$ which is analytic in $\mathbb{C} \backslash\{1\}$ with a simple pole of residue 1 at $s=1$.

Remark 5.1. We notice that in the case $\alpha>1$, we may write

$$
E_{\alpha}(s)=(\alpha-1)^{-s}+\frac{1}{2 \pi i} \int_{\eta_{\alpha}^{\prime}} \frac{\zeta^{\prime}(w)}{\zeta(w)}(\alpha-w)^{-s} \mathrm{~d} w
$$

where the modified contour $\eta_{\alpha}^{\prime}$ stays to the right of the pole at 1 . We may then substitute the Dirichlet series for $\zeta^{\prime}(s) / \zeta(s)$ in the latter integral and obtain the representation (see [Vor10, formula (7.28)])

$$
\zeta_{\alpha}(s)=(\alpha-1)^{-s}-\frac{1}{\Gamma(s)} \sum_{n=2}^{\infty} \frac{\Lambda(n)(\log n)^{s-1}}{n^{\alpha}}-2^{-s} \zeta(s, \alpha / 2+1),
$$

\footnotetext{
${ }^{6}$ We urge the reader to take a look at Frankenhuijsen's MathSciNet review of the book, where Serge Lang's point of view of "climbing the ladder" is explained.
} 
as can be seen by employing the formula

$$
\frac{1}{2 \pi i} \int_{\eta_{\alpha}} n^{-w}(\alpha-w)^{-s} \mathrm{~d} w=\frac{-(\log n)^{s-1}}{n^{\alpha} \Gamma(s)} .
$$

To verify (5.32), we may first assume that $0<s<1$ since the general case then follows by analytic continuation. When $0<s<1$, we may push the integral to the interval $[\alpha, \infty)$, which produces an additional factor of $-2 \sin (\pi s)$. The integral then reduces to the definition of $\Gamma(1-s)$, and the rest follows by Euler's reflection formula

$$
\sin (\pi s)=\frac{\pi}{\Gamma(s) \Gamma(1-s)} .
$$

We note also that other functions than $\rho^{-s}$ may be chosen. As an example, we may take $F(s):=$ $\exp \left((\alpha-s)^{c}\right)$ with $0<c<1$. Then a modification of the above augment verifies that the "zeta function"

$$
Z_{F}(s):=\sum_{\rho} F(\rho)^{-s}
$$

has an analytic extension to $\mathbb{C}$.

\section{References}

[Chak70] I. C. Chakravarty, The secondary zeta-functions, J. Math. Anal. Appl. 30 (1970), $280-294$.

[Chak71] I. C. Chakravarty, Certain properties of a pair of secondary zeta-functions, J. Math. Anal. Appl. 35 (1971), $484-495$.

[Del66] J. Delsarte, Formules de Poisson avec reste, J. Analyse Math. 17 (1966), 419-431.

[Fu83] A. Fujii, The zeros of the zeta function and Gibbs's phenomenon, Comment. Math. Univ. Sancti Pauli 32 (1983), $229-248$.

[Fu02] A. Fujii, On the zeros of the Riemann zeta function, Comment. Math. Univ. St. Paul. 51 (2002), 1-17.

[Gar07] J. Garnett, Bounded Analytic Functions, Revised first edition, Graduate Texts in Mathematics 236, Springer, New York, 2007.

[Gho81] A. Ghosh, On Riemann's zeta function-sign changes of $S(T)$, Recent progress in analytic number theory, Vol. 1 (Durham, 1979), pp. 25-46, Academic Press, London-New York, 1981.

[Iv03] A. Ivić, The Riemann Zeta-Function. Theory and Applications, Reprint of the 1985 original [Wiley, New York], Dover Publications, Inc., Mineola, NY, 2003.

[Iv01] A. Ivić, On certain sums over ordinates of zeta-zeros, Bull. Cl. Sci. Math. Nat. Sci. Math. 26 (2001), $39-52$.

[Mel17] Hj. Mellin, Über die nullstellen der Zetafunktion, Ann. Acad. Sci. Fenn. A 10 no. 11 (1917).

[MoVa74] H. L. Montgomery and R. C. Vaughan, Hilbert's inequality, J. Lond. Math. Soc. 8 (1974), 73-82.

[Sel43] A. Selberg, On the normal density of primes in small intervals, and the difference between consecutive primes, Arch. Math. Naturvid. 48 (1943), 87-105.

[Sp76] R. Spira, Zeros of Hurwitz zeta functions, Math. Comp. 30 (1976), 863-866.

[Ten95] G. Tenenbaum, Introduction to Analytic and Probabilistic Number Theory, Cambridge Studies in Advanced Mathematics 46, Cambridge University Press, Cambridge, 1995.

[Ti86] E. C. Titchmarsh, The Theory of the Riemann Zeta-Function, 2nd Edition, Oxford University Press, New York, 1986.

[Vor10] A. Voros, Zeta Functions over Zeros of Zeta Functions, Lecture Notes of the Unione Matematica Italiana 8, SpringerVerlag, Berlin; UMI, Bologna, 2010. 


\section{Andriy Bondarenko}

Department of Mathematical Sciences

Norwegian University of Science and Technology (NTNU)

NO-7491 Trondheim, Norway

e-mail: andriybond@gmail.com

\section{Aleksandar Ivić}

Serbian Academy of Sciences and Arts

Knez Mihailova 35

11000 Beograd, Serbia

e-mail: aleksandar.ivic@rgf.bg.ac.rs

\section{Eero Saksman}

Department of Mathematical Sciences

Norwegian University of Science and Technology (NTNU)

NO-7491 Trondheim, Norway

and

University of Helsinki

Department of Mathematics and Statistics, P.O. Box 68

FIN-00014 University of Helsinki, Finland

e-mail: eero.saksman@helsinki.fi

\section{Kristian Seip}

Department of Mathematical Sciences

Norwegian University of Science and Technology (NTNU)

NO-7491 Trondheim, Norway

e-mail: kristian.seip@ntnu.no 